\title{
Preliminary Study of Wide Base-of-Support Footwear in Preventing Falls Caused by Lateral Slip during Walking
}

\author{
Takeshi Yamaguchi $^{1) *}$, Masaru Yano ${ }^{1)}$, Shinya Fukuzawa ${ }^{1)}$, Hiroshi Onodera ${ }^{2)}$ and Kazuo Hokkirigawa ${ }^{1)}$ \\ ${ }^{1)}$ Graduate School of Engineering, Tohoku University \\ 6-6-01 Aramaki-Aza-Aoba, Aoba-ku, Sendai, Miyagi 980-8579, Japan \\ 2) Japan Science and Technology Agency (JST)-CREST \\ 2-11-11 Kagitori-Honcho, Taihaku-ku, Sendai, Miyagi 982-8555, Japan \\ *Corresponding author: yamatake@gdl.mech.tohoku.ac.jp
}

( Manuscript received 3 January 2012; accepted 1 March 2012; published 9 September 2012 )

(Presented at Technical Session in the International Tribology Conference Hiroshima 2011)

\begin{abstract}
The present preliminary study examined whether an increase in the lateral width of footwear (base-of-support width) can reduce the risk of falling caused by induced lateral slip while turning on slippery surfaces. Wide base-of-support (BOS) footwear was designed and manufactured, with lateral widening achieved by fixing a BOS extension, using a planar linkage of 70-mm width on the lateral side of normal BOS footwear. Gait trials were conducted on 16 young healthy male adults. Subjects were instructed to walk in a straight line and to turn $60^{\circ}$ to the right on a stainless steel floor covered with glycerol solution. As compared with the normal BOS footwear, the wide BOS footwear reduced the frequency of trials in which fall occurred due to lateral slip by up to $67 \%$ and the slip distance and slip velocity of the supporting foot were reduced by up to $40 \%$ and $35 \%$, respectively. The results demonstrated the efficacy of increased lateral BOS width in reducing the risk of fall caused by induced lateral slip.
\end{abstract}

Keywords: slip, fall, base-of-support, footwear, friction

\section{Introduction}

In Japan, falling accidents have recently become an increasingly serious problem with the mortality rate exceeding that caused by traffic accidents [1], and slipping is one of the leading causes of falling accidents [2-6]. Slipping is likely to occur during transient movements such as turning, in which walking speed and direction are changed, compared with steady-state movement such as linear walking at constant speed. This slipping results due to increased traction coefficient at the shoe-floor interface [7-10]. When slip occurs during turning, the supporting foot slides laterally, in the direction opposite to turning, causing a lateral fall [11]. For elderly people, such lateral falls increase the risk of debilitating and life-threatening femoral neck or hip fractures [12].

In order to maintain postural stability, the displacement and velocity of the body center of mass (COM) must be regulated with respect to the base-of-support (BOS) defined by the feet [13-15]. When standing still, the COM should be located over a stationary BOS to control postural stability. On the other hand, when lateral slip occurs during single support periods during
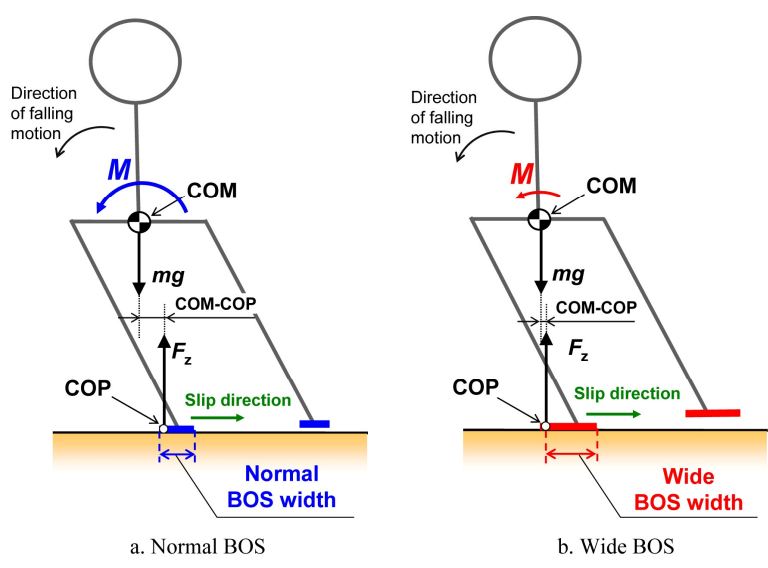

Fig. 1 Schematic of the positional relationship of the COM and BOS in the frontal plane when lateral slip (to the right) occurs with the right foot. $M$, moment on the COM; $m$, whole body mass; $g$, gravity; $F_{\mathrm{z}}$, vertical ground reaction force. 
walking, the supporting foot moves laterally and the COM may be outside the BOS as shown in Fig.1a. Successful balance recovery, by means of (compensatory) stepping, requires foot movement to arrest the COM within the boundaries of the new BOS established by the compensatory step $[14,16]$. However, the slip, which creates a greater distance between the COM and BOS edge, will lead to larger moment acting on the COM in the falling direction caused by the greater moment arm length in the lateral direction (lateral distance between the COM and the center of pressure (COP) of the supporting foot). The COP corresponds to the acting point of the ground reaction force.

It may be effective to increase the lateral BOS width to reduce the risk of falling caused by lateral slip, because increased BOS width would easily restrict the $\mathrm{COM}$ within the boundaries of the BOS. Furthermore, because the COP can be located more laterally, any increase in the BOS width would reduce the moment on the COM in the falling direction by shortening the lateral moment arm length (lateral COM-COP difference), as shown in Fig. 1b. Increased BOS width would also provide a time margin for the COP to reach the lateral boundary of the BOS, which would render execution of the lateral step easier. However, an excessive increase in the BOS width could affect the gait and agility during walking or stepping, resulting in foot collision or trip.

The objective of this study was to determine whether increased BOS width would reduce the risk of falling caused by lateral slip during walking. In the preliminary phase, we examined the risk of fall on a slippery floor in young healthy male adults during turning with both normal BOS footwear and laterally widened (by $70 \mathrm{~mm}$ ) BOS footwear (wide BOS footwear, which was specially designed for this study).

\section{Methods}

\subsection{Preparation of wide BOS shoes}

Figure 2 shows normal BOS footwear and wide BOS footwear, which was specially designed for this study. The BOS extension, using planar linkage with stainless steel plates, was fixed to the lateral aspect of commercially available footwear (normal BOS footwear, length: $270 \mathrm{~mm}$ ) to increase the lateral BOS width. The width of the BOS extension was set at $70 \mathrm{~mm}$. A steel plate was inserted beneath the insole of the normal footwear to equate the weight of the wide BOS footwear, in order to eliminate the effect of weight difference. The weight of each shoe was approximately $1 \mathrm{~kg}$. Nitrile butadiene rubber (NBR) sheet (thickness: $1.8 \mathrm{~mm}$ ) was affixed to the outer sole of each shoe, and an NBR block (thickness: $10 \mathrm{~mm}$ ) was also affixed to the sole of the BOS extension. None of the subjects needed packing to fill any space between the shoe and foot.

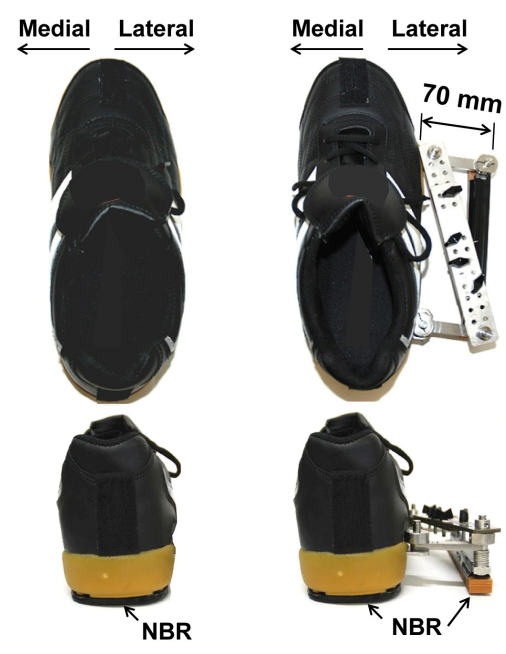

Fig. 2 Footwear used in this study; a) Normal BOS footwear (left), b) Wide BOS footwear (right)

\subsection{Subjects}

The study comprised 16 healthy male adults with an average age of 21.4 years (range: 21-26). Mean \pm standard deviation for height and weight of the subjects were $1.74 \pm 0.06 \mathrm{~m}$ and $62.4 \pm 6.4 \mathrm{~kg}$, respectively. This study was approved by the Institutional Review Board of National Nishitaga Hospital, Japan, and informed consent was obtained from all subjects.

\subsection{Experimental procedure}

Figure 3 shows schematic diagrams of the experimental set-up and movement instructions given to subjects in this study. Gait trials were performed on a 5 -m long walkway. Four force plates were installed in the center of the walkway and ground reaction forces were collected at a sampling rate of $60 \mathrm{~Hz}$. Stainless steel flooring $(2 \mathrm{~m} \times 1 \mathrm{~m} \times 2 \mathrm{~mm})$, polished with \#400
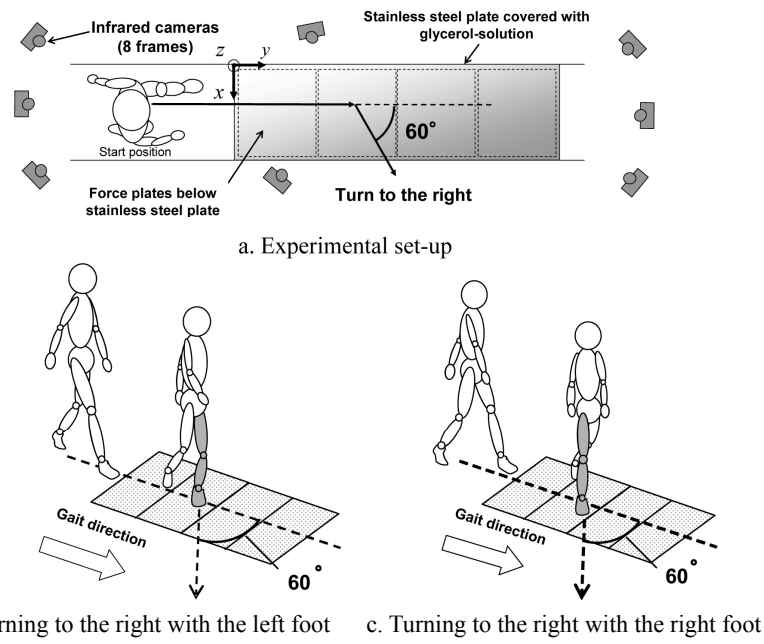

Fig. 3 Schematic diagrams of the experimental set-up and movement instructions to subjects (turning $60^{\circ}$ to the right) 
abrasive paper, was mounted on the walkway and covered with a solution of glycerol (70 wt.\%; viscosity: $19.7 \mathrm{mPa} \cdot \mathrm{s})$. An eight-camera motion measurement system (Motion Analysis Corporation, MAC3D System) recorded three-dimensional motion data at a sampling rate of $60 \mathrm{~Hz}$ from 25 reflective markers placed on the body and footwear (toe and heel), based on the Helen Hayes marker set [17].

In order to induce the lateral slip, subjects were instructed to walk in a straight line and to turn $60^{\circ}$ to the right with the left or right foot landing on the stainless steel surface, as shown in Fig. 3. Subjects were tested with the normal and wide BOS footwear in separate sessions. The order of testing for the two footwear conditions was balanced across subjects to eliminate the effect of testing order of footwear on the results. Replication of trials for similar conditions was 6 (total: 24 trials for each subject). Subjects were instructed to walk as naturally as possible at a self-selected pace, with a straight graze and to "do whatever comes naturally to prevent yourself from falling." Subjects wore a safety harness that was designed to prevent the impact with the floor without otherwise restricting movement that could aid in the control of balance, as shown in Fig. 4. All trials were videotaped.

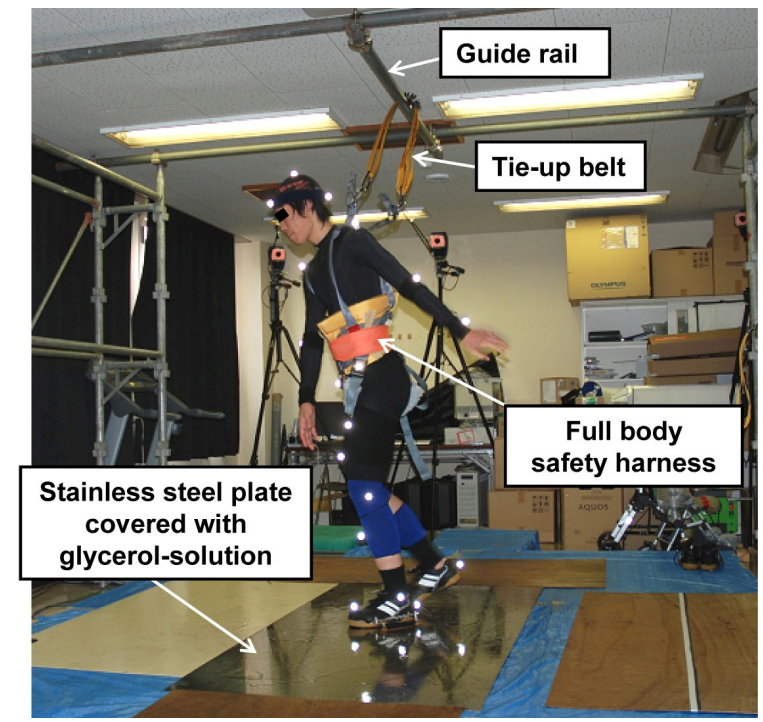

Fig. 4 Photograph of gait trials on stainless steel floor covered with glycerol solution

\subsection{Data analysis}

Frequency of trials in which fall occurred was examined for both types of footwear. We defined a "fall" as subjects' feet being off the floor, followed by the subjects being completely suspended in the harness after losing balance due to slip. Whether subjects had fallen was identified on video data. Vertical ground reaction force $F_{\mathrm{z}}$ data collected from the force plates were also used to determine whether both of subjects' feet were off the floor $\left(F_{\mathrm{z}}=0\right.$ if both feet were off the floor).

Slip distance and slip velocity (sliding velocity of the supporting foot) were calculated for each trial, using the position data from a reflective marker attached to the toe of the footwear, to examine slip severity. The co-ordinate data of reflective markers were digitally smoothed using a two-order low-pass Butterworth filter with a cut-off frequency of $6 \mathrm{~Hz}$. Slip distance $(D)$ was calculated according to the following formula;

$$
\begin{aligned}
D & =\sum_{i=m}^{n-1} D(i) \\
& =\sum_{i=m}^{n-1} \sqrt{\left(x_{t o e}(i+1)-x_{t o e}(i)\right)^{2}+\left(y_{t o e}(i+1)-y_{t o e}(i)\right)^{2}}
\end{aligned}
$$

where, $i$ is the frame number, $m$ is the frame number at slip initiation, $n$ is the frame number at slip termination, and $x_{\text {toe }}(\mathrm{i})$ and $y_{\text {toe }}(\mathrm{i})$ are the co-ordinates of the toe marker.

The velocity of the toe marker in the $x$ and $y$ directions, $v_{\text {toe_x }}(\mathrm{i})$ and $v_{\text {toe } y}(\mathrm{i})$, respectively, was calculated according to the following formulae:

$$
\begin{aligned}
& v_{\text {toe_x }}(i)=60\left(x_{\text {toe }}(i+1)-x_{\text {toe }}(i)\right) \\
& v_{\text {toe_y }}(i)=60\left(y_{\text {toe }}(i+1)-y_{\text {toe }}(i)\right)
\end{aligned}
$$

thus,

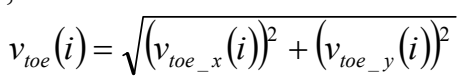

The maximum value of the velocity of the toe marker was used as the maximum slip velocity $v_{\max }$ for the analysis.

Paired t-test was used to identify the differences in the mean values of frequency of trials with fall, slip distance, and maximum slip velocity for both types of footwear. Significance level was set at 0.05 . Analysis was performed using SPSS (version 19.0, Chicago, IL, USA).

\section{Results and discussion}

Figure 5 shows the mean frequency of trials in which fall occurred with both types of footwear when turning with the left foot (Fig. 5a) and right foot (Fig. $5 \mathrm{~b}$ ). Error bars mean standard deviations. The mean values of frequency of fall trials for the normal BOS footwear when turning with the left and right feet were $41.7 \%$ (40/96) and 46.9\% (45/96), respectively. In contrast, the mean values of the frequency of fall trials for the wide BOS footwear when turning with the left and right feet were 18.8\% (18/96) and 15.6\% (15/96), respectively. The wide BOS footwear reduced the frequency of fall trials by $55 \%$ and $67 \%$ for turning with the left and right feet, respectively, compared with the normal BOS footwear $(p<0.05)$. Therefore, it was confirmed that the wide BOS footwear can reduce the risk of falls caused by induced lateral slip when turning on slippery flooring.

There was no significant difference in the mean 
friction coefficient of the supporting foot and the floor during slipping (calculated from the ground reaction forces) between the normal BOS footwear $(0.038 \pm$ 0.017 for the left foot pivoting; $0.034 \pm 0.08$ for the right foot pivoting) and the wide BOS footwear $(0.041 \pm$ 0.026 for the left foot pivoting; $0.033 \pm 0.01$ for the right foot pivoting) $(p>0.05)$.
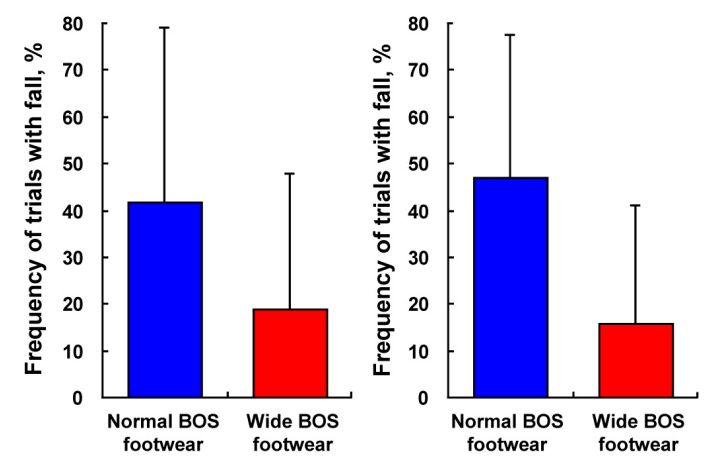

a. Turning with the left foot

b. Turning with the right foot

Fig. 5 Frequency of trials in which fall occurred

Increased slip distance and slip velocity of the supporting foot could increase the relative distance between the COM and COP, resulting in loss of postural stability due to an increased moment on the COM in the falling direction. Thus, it has been pointed out that slip distance and slip velocity highly correlate with the risk of falling [18]. Figure 6 shows the relationship between mean values for slip distance and maximum slip velocity of the sliding foot. Error bars mean standard deviations. When turning to the right with the left foot (Fig. 6a), the wide BOS footwear reduced the mean value of slip distance and mean value of the maximum slip velocity by $22.8 \%$ and $27.5 \%$, respectively, compared with the normal footwear $(p<0.05)$. When turning to the right with the right foot (Fig. 6b), the wide BOS footwear also reduced the mean value of slip distance and mean value of maximum slip velocity by
$40.0 \%$ and $34.7 \%$, respectively $(p<0.05)$.

Figures 7 and 8 show successive images of typical trials in which a fall occurred when turning to the right with the left and right feet, respectively. As shown in Fig. 7, lateral slip (to the left) occurred at the pivoting left foot, and then the subject stepped with the right foot to recover the balance. However, a large lateral slip (to the left) again occurred at the right foot, resulting in a lateral fall. On the other hand, when turning to the right with the right foot, a large lateral slip (to the left) occurred at the pivoting right foot, the subject then being unable to make a compensatory step with the left foot resulted in a fall as shown in Fig. 8. As is indicated in Figs. 7 and 8, falls were likely to occur due to a large lateral slip (to the left) of the right foot. When a large lateral slip of the right foot occurs, the moment on the $\mathrm{COM}$ in the falling direction increases because the lateral distance between the COM and COP (within the BOS) increases. This would possibly produce difficulty in cross-over stepping with the left foot to recover balance. The wide BOS footwear reduced slip velocity and slip distance as shown in Fig. 6, and therefore the horizontal distance between the COM and lateral BOS edge of the right foot decreased. In addition, the COP may be located more laterally with the wide BOS footwear compared with the normal BOS footwear. Thus, the moment acting on the COM in the falling direction would be reduced by shortening the length of the moment arm in the lateral direction, which would result in a reduction in the frequency of fall with or without compensatory stepping with the left foot.

In this study, it was demonstrated that wide BOS footwear reduces the risk of falling caused by lateral slip when turning. However, the weight of the footwear was approximately $1 \mathrm{~kg}$ and the width of the lateral extension of the BOS was large $(70 \mathrm{~mm})$; these factors may have affected the gait and agility. In future studies, an optimum (minimum) width for the lateral extension of the BOS, which has the ability to reduce the risk of slip and fall but affects gait and agility less, will be clarified. In addition, a reduction in the weight of the

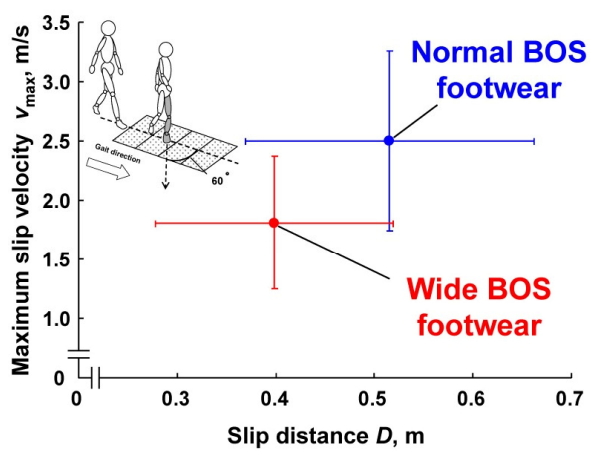

a. Turning with the left foot

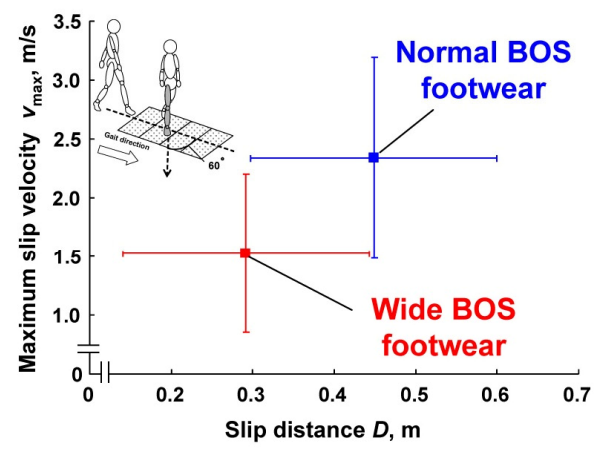

b. Turning with the right foot

Fig. 6 Slip distance and maximum slip velocity 


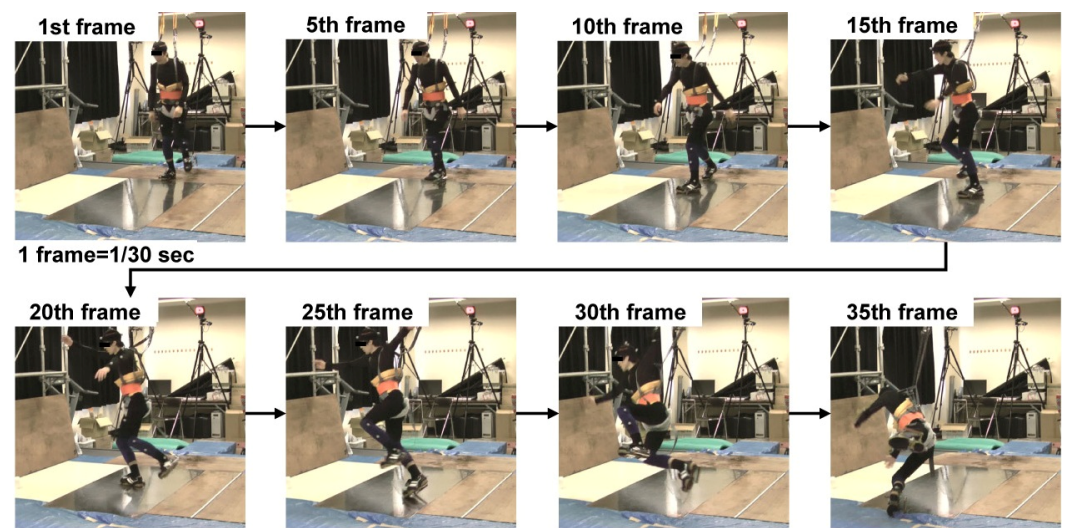

Fig. 7 Successive images of trials in which fall occurred when turning with the left foot

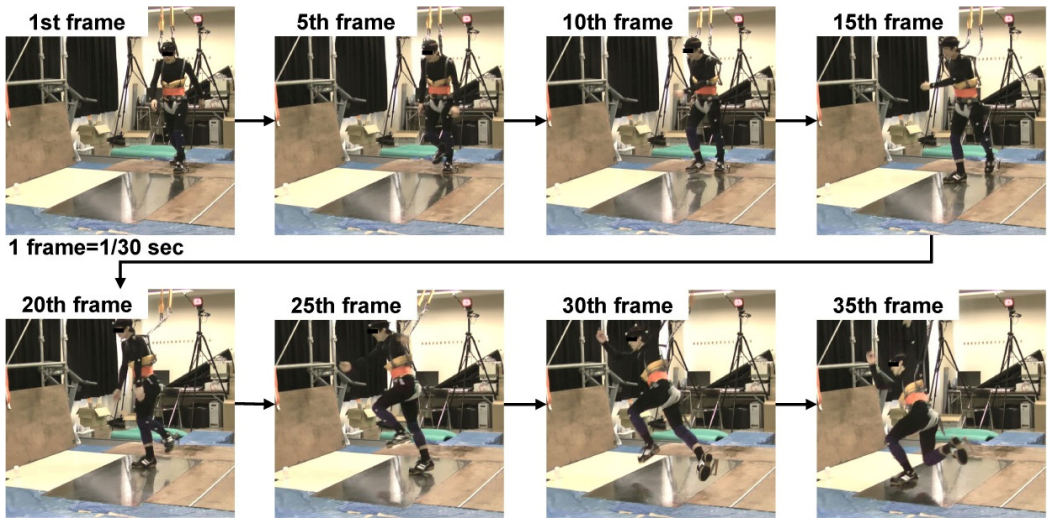

Fig. 8 Successive images of trials in which fall occurred when turning with the right foot

footwear will be sought. This study involved only young healthy adults. It would also be necessary to investigate whether wide BOS footwear is effective in older adults to reduce the risk of fall due to slipping.

\section{Conclusions}

The present study examined whether wide BOS footwear, in which the BOS was extended by $70 \mathrm{~mm}$ laterally, reduces the risk of fall caused by lateral slip during turning on a slippery floor. The conclusions are summarized as follows:

1) Wide BOS footwear reduced the frequency of fall trials because of lateral slip by $55 \%$ and $67 \%$ for turning with the left and right feet, respectively, compared with normal BOS footwear.

2) When turning to the right with the left foot, wide BOS footwear reduced slip distance and maximum slip velocity during slipping by $22.8 \%$ and $27.5 \%$, respectively, compared with normal BOS footwear.

3) When turning to the right with the right foot, wide BOS footwear reduced slip distance and maximum slip velocity during slipping by $40.0 \%$ and $34.7 \%$, respectively, compared with normal BOS footwear.

\section{Acknowledgement}

This work was supported by Grant-in-Aid for Young Scientists (B) (22760110) from the Japan Society for the Promotion of Science (JSPS). This study was supported in part by Core Research for Evolutional Science and Technology (CREST) Program of Japan Science and Technology Agency (JST).

\section{References}

[1] Statistics and Information Department, Minister's Secretariat, Ministry of Health, Labour and Welfare, "Vital Statistics of Japan 2009," Health and Welfare Statistics Association, Tokyo, 3, 2011.

[2] Courtney, T. K., Sorock, G. S., Manning, D. P., Collins, J. W. and Holbein-Jenny, M. A., "Occupational Slip, Trip, and Fall-Related Injuries - Can the Contribution of Slipperiness Be Isolated?," Ergonomics, 44, 2001, 1118-1137.

[3] Lloyd, D. G. and Stevenson, M. G., "Investigation of Floor Surface Profile Characteristics that Will Reduce the Incidence of Slips and Falls," 
Mechanical Engineering Transaction Institution of Engineers (Australia), ME17, 2, 1992, 99-104.

[4] Strandberg, L. and Lanshammar, H., "The Dynamics of Slipping Accidents," Journal of Occupational Accidents, 3, 1981, 153-162.

[5] Cohen, H. H. and Compton, D. M. J., "Fall Accident Patterns: Characterization of Most Frequent Work Surface-Related Injuries," Professional Safety, 27, 1982, 16-35.

[6] Nordin, M., Andersson, G. and Pope, M., "Musculoskeletal Disorders in the Workplace," Moseby -Year Inc., St Louis, 1997, 152-166.

[7] Yamaguchi, T. and Hokkirigawa, K., “"Walking-Mode Maps' Based on Slip/Non Slip Criteria," Industrial Health, 46, 1, 2008, 23-31.

[8] Yamaguchi, T., Hatanaka, S. and Hokkirigawa, K., "Effect of Step Length and Walking Speed on Traction Coefficient and Slip between Shoe Sole and Walkway," Tribology Online, 3, 2, 2008, 59-64.

[9] Yamaguchi, T., Yamanouchi, H. and Hokkirigawa, K., "Experimental Analyses of Load Carrying Effects on the Peak Traction Coefficient between Shoe Sole and Floor during Walking," Tribology Online, 3, 6, 2008, 342-347.

[10] Yamaguchi, T., Yano, M., Nagamori, K., Onodera, H. and Hokkirigawa, K., "Experimental Analysis of Slip and Fall during Transient Walking," Proc. JAST Tribology Conference, Fukui, September 2010, 427-428 (in Japanese).

[11] Smeesters, C., Hayes, W. C. and McMahon, T. A., "Disturbance Type of Gait Speed Affect Fall
Direction and Impact Location," Journal of Biomechanics, 34, 2001, 309-317.

[12] Cummings, S. R. and Nevitt, M. C., "Non-Skeletal Determinants of Fractures: the Potential Importance of the Mechanics of Falls," Osteoporosis International, 1, 1994, 67-70.

[13] Pai, Y. C. and Patton, J., "Center of Mass Velocity-Position Predictions for Balance Control," Journal of Biomechanics, 30, 1997, 347-354.

[14] Maki, B. E. and McIlroy, W. E., "The Control of Foot Placement during Compensatory Stepping Reactions: Does Speed of Response Take Precedence over Stability?," IEEE Transactions on Rehabilitation Engineering, 7, 1999, 80-90.

[15] Maki, B. E., McIlroy, W. E. and Perry, S. D., "Influence of Lateral Destabilization on Compensatory Stepping Responses," Journal of Biomechanics, 29, 1996, 343-353.

[16] Maki, B. E. and McIlroy, W. E., "Control of Compensatory Stepping Reactions: Age-Related Impairment and the Potential for Remedial Intervention," Physiotherapy Theory Practice, 15, 1999, 69-90.

[17] Kadaba, M. P., Ramakrishnan, H. K. and Wootten M. E., "Measurement of Lower Extremity Kinematics During Level Walking," Journal of Orthopaedic Research, 8, 1990, 383-392.

[18] Bhatt, T., Wening, J. D.and Pai, Y.-C., "Influence of Gait Speed on Stability: Recovery from Anterior Slips and Compensatory Stepping," Gait and Posture, 21, 2005, 146-156. 